\title{
The curative effect of the associated cell transplantation on the rabbit myocardial infarction
}

\author{
Zhicheng Fang $^{1^{*}}$, Chang'e Zhou ${ }^{2}$, Xiang Zheng $^{1}$, Boyi Liu ${ }^{1}$, Li Chen ${ }^{1}$, Chunfeng Shen $^{1}$, \\ Pei Liu ${ }^{1}$, Yunfei Huang ${ }^{1}$ \\ ${ }^{1}$ Department of Intensive Care Unit, Taihe Hospital, Hubei Medicine University, Shiyan, China; \\ *Corresponding Author: 13593751009@139.com \\ ${ }^{2}$ Department of Nephrology, Taihe Hospital, Hubei Medicine University, Shiyan, China
}

Received 10 May 2013; revised 10 June 2013; accepted 20 June 2013

Copyright (C) 2013 Zhicheng Fang et al. This is an open access article distributed under the Creative Commons Attribution License, which permits unrestricted use, distribution, and reproduction in any medium, provided the original work is properly cited.

\section{ABSTRACT}

Inducing Mesenchymal stem cells to differentiate into cardiomyocycte-like cells and endothelial progenitor cells orientedly and evaluating the curative effect of the associated cell transplantation on the rabbit myocardial infarction (MI). Methods: Mesenchymal stem cells (MSCs) were isolated from the bone marrow of 24 rabbits and cultured in special cell culture medium containing 5-azacytidine (5-AZA), endothelial cell growth supplements (ECGS), vascular endothelial growth factor (VEGF) and basic fibroblast growth factor (BFGF) respectively. The cell transplantation was performed 2 weeks after MI. Rabbits were divided into control group, cardiomyocytes-like cell group, endothelial progenitor cell group and combination group. We used the echocardiography to measure the heart function 2 to 4 weeks after MI, TTC to measure the area of the infarction, flow cytometry to estimate the cell apoptosis. Results: After induced, MSCs were differentiated orientedly into cardiomyocycte-like cells (CLCs) and endothelial progenitor cells (EPCs). CLCs became greater and had a "stick" or "ball" shape. Transmisson electron microscopy showed that the cells had oval nuclei positioned in the central part and well organized myofilaments, atrial granules and mitochomdrion. RT-PCR showed the expression of the atrial natriuretic polypeptide, phospholamban and myosin heavy chain in CLCs. EPCs formed confluent one-celled layer which showed a cobblestone shape by phase-contrast microscope. The expression of CD133 in EPCs was much at first and then descended gradually. Compared with the control group, cell transplantation could im- prove the heart function, reduce the size of MI, decrease the left ventricular end systole diameter and end diastolic diameter, suppressed cell apoptosis. The curative effect of cell transplantation was better in the associated-cell group than in the single-cell transplantation group (LVEF: $32.49 \% \pm 1.29 \%$ vs $53.22 \% \pm 2.13 \%$ vs $56.91 \% \pm$ $2.04 \%$ vs $62.61 \% \pm 2.37 \%, P<0.05$; LVESD: 1.23 \pm 0.02 vs $0.98 \pm 0.04$ vs $0.98 \pm 0.12$ vs $1.11 \pm 0.03$, $P<0.05$; LVEDD: $1.53 \pm 0.13$ vs $1.24 \pm 0.02$ vs $1.21 \pm 0.09$ vs $1.01 \pm 0.01, P<0.05$; the area of infarction: $35.17 \% \pm 0.98 \%$ vs $28.61 \% \pm 1.24 \%$ vs $29.73 \% \pm 2.11 \%$ vs $22.82 \% \pm 3.12 \%, P<0.05$; apotosis: $8.6 \% \pm 0.94 \%$ vs $6.94 \% \pm 0.59 \%$ vs $6.4 \% \pm$ $0.27 \%$ vs $4.63 \% \pm 0.74 \%, P<0.05)$. Conclusions: This study showed that MSCs can differentiate into CLCs and EPCs in the given conditions and the associated cell transplantation is better than the single cell transplantation to treat MI.

Keywords: Mesenchymal Stem Cell; Cardiomyocycte-Like Cell; Endothelial Progenitor Cell; Cell Transplantation

\section{INTRODUCTION}

The prognosis of the myocardial infarction (MI) has been improved greatly by medication and intervention therapy, but there are still many problems to be resolved. Nowadays, lots of studies show that cell transplantation has positive effect on treating MI. Cardiomyocytes-like cells and endothelial progenitor cells are the two main kinds of cells used for transplantation. Because the former is used for myocardial regeneration and the latter for myocardial ischemia, if we transplanted only one kind of cell, the therapeutic effect is limited. In this study, we explore whether the associated cells transplantation is 
superior to the single cell transplantation $[1,2]$.

\section{METHODS}

\subsection{Experimental Animals and Cell Transplantation}

Ten Japanese big-ear rabbits, including both male and female, weighing $2-2.5 \mathrm{~kg}$, were anesthetized with $10 \%$ urethane. Additional smaller doses were given as needed to maintain deep anesthesia. Under controlled ventilation, a thoracotomy through a left parasternal incision was performed, the pericardium was incised and the anterior wall of the left ventricle was exposed. The left anterior descending (LAD) coronary artery of the animals was identified and carefully separated, then ligated downstream $2 \mathrm{~mm}$ from where the first diagonal artery branches out. After successfully producing an anterior MI, which was confirmed by elevation of the ST segment by more than $0.2 \mathrm{mV}$ in leads I, II and aVL, the rabbits were randomized into four groups, the control group (Cont group), the cardiomyocycte-like cell group (CLC group), the endothelial progenitor cell group (EPC group) and the combination group (Comb group). All the rabbits underwent another thoracotomy after 2 weeks of MI. We identified the necrotic muscle which was pale and had less motion, and then injected $100 \mu \mathrm{L}$ cell suspensions into six random points at the edge of the necrosis. The Cont group was given saline instead of cell suspension. The cell suspension contained $5 \times 10^{7}$ cells of CLCs, EPCs, or both of them, which had been marked by Brdu before cell transplantation. The total number of cells injected into per rabbit was $3 \times 10^{8}$. Postoperatively, each rabbit received penicillin and gentamicin intramuscularly daily for 7 days, and were treated kindly.

\subsection{Mesenchymal Stem Cells (MSCs) Isolation}

Bone marrow $(4-5 \mathrm{~mL})$ was extracted from ilium, moved into Percoll lysis, and centrifuged $(1500 \mathrm{rpm}, 20$ $\mathrm{min})$. The white homogenate with mononuclear cells was moved into another centrifuge tube, washed with DMEM culture medium and centrifuged twice $(2000 \mathrm{rpm}, 5 \mathrm{~min})$. After that, the cell precipitation $(5 \mathrm{~mL})$ was reserved, cultured in the high- and low-sugar DMEM culture medium and blowed evenly. Then the cells were inoculated into culture dish $\left(2 \times 10^{6}\right.$ cells per dish $)$ and ventilated with $5 \% \mathrm{CO}_{2}$ at $37^{\circ} \mathrm{C}$. After $3-4$ days, the suspended cells were discarded. 5-azacytidine (5-AZA), endothelial cell growth supplements (ECGS), vascular endothelial growth factor (VEGF) and basic fibroblast growth factor (BFGF) were added into the dishes respectively, so as to induce MSCs into CLCs and EPCs. Change the culture medium every 2 or 3 days and observe the cells every day.

\subsection{Cardiomyocycte-Like Cells (CLCs) Detection}

After adding 5-AZA, we used the phase-contrast microscope to observe the changes of the cell shape every day and used the electron microscope to observe the ultrastructure of CLCs. We also used RT-PCR to analyze the expression of $\beta$-myosin heavy main $(\beta$-MHC), phospholamban (PHO) and atrial natriuretic polypeptide (ANP) quantificationally.

\subsection{Endothelial Progenitor Cells (EPCs) Detection}

After adding ECGS, VEGF and BFGF, we used the phase-contrast microscope to observe the changes of the cell shape every day and used the flow cytometry to detect the expression of CD133 every week. The cells were reserved after digested with $0.25 \%$ trypsin and discarded the suspension. Mix the cells with $1 \mathrm{~mL}$ PBS, filter them by nylon web and centrifuge it at $1000 \mathrm{rpm}$ for $5 \mathrm{~min}$. Then reserve the precipitation and add $20 \mu \mathrm{L}$ CD133 FITD into it. Finally, we used the flow cytometry to demonstrate the expression rate of CD133 which was showed by the number of the fluorescent cells per 1000 cells.

\subsection{Evaluating the Heart Function and Ventricle Remodeling}

Echocardiography was performed to assess viable myocardium via parasternal long-axis and short-axis views by a veteran doctor. We measured the left ventricular end systole diameter (LVESD), the left ventricular end diastolic diameter (LVEDD), the left ventricular posterior wall hypertrophy (LVPWH), the fractional shortening (FS), the stork volume (SV) and the left ventricular ejection fraction (LVEF).

\subsection{Evaluating the Area of $\mathrm{Ml}$ and the Apoptosis}

After 4 weeks of cell transplantation, we executed the rabbits and took out of the hearts. We cut down the left ventricle and put it into the refrigerator at $-80^{\circ} \mathrm{C}$ for $3-5$ $\min$. Then we incised the left ventricle into $2 \mathrm{~mm}$ wide slices along the axis from apex to base. The slices were put into the $2 \%$ TTC solution and dyed for $30 \mathrm{~min}$ at $37^{\circ} \mathrm{C}$. Using the image analysis system to measure the area of the infarction and calculate the area rate in each slice. Repeating 3 times and calcutating the average as the area of MI. 3 plots $(5 \times 5 \mathrm{~mm})$ were cut from the infarction, washed with the saline and cut into small pieces. digest with $0.25 \%$ trypsin and discard the suspension. Mix the left cell precipitation with $1 \mathrm{~mL}$ PBS, filter and centrifuge (1000 rpm, $5 \mathrm{~min}$ ). Then reserve the cell pre- 
cipitation again. We added DNA-PrepTM LPR into it and placed it for $1 \mathrm{~min}$, then added DNA-PrepTM Stain and placed it for $15-20 \mathrm{~min}$ avoiding the light. We measured the content of the hypodiploid DNA in the total DNA as the apoptosis, repeating 3 times and calculating the average.

\section{STATISTICAL ANALYSIS}

Continuous variables are presented as mean \pm SD. Statistical comparisons among the four groups were made by ANOVA and q test. Statistical computing was performed by use of SPSS (Version 10.0, SPSS Inc.); for all tests, a probability level of $P<0.05$ was considered significant.

\section{RESULTS}

\subsection{The Morphous of Cardiomyocycte-Like Cells (CLCs) and Endothelial Progenitor Cells (EPCs)}

MSCs became bigger after induced by 5-AZA and showed the shape of "short stick" or "pearl" (Figures 1(a) and (b)). After 2 weeks, the cells syncretized and fusiformly arrayed with directionality. After culturing 3 weeks, the conjunctions among the cells were abundant and the myotube structure appeared, but spontaneous beats were not found. MSCs, induced by ECGS et al., proliferated rapidly after a short period of retention. Within 1 - 2 days, the cells became fusiform and cluster. After 16 - 18 days, the cells were nearly syncretized completedly and showed the shape of cobblestone shope or slabstone (Figure 2(a)).

\subsection{The Indentification of CLCs and EPCs in Vitro}

Observed by transmission electron microscope, CLCs had one orbicular-ovate nuclear locating in the center and contained lots of mitochondria. ANPs were at the surrounding of the nuclear with high electron density and its diameter was $200-300 \mu \mathrm{m}$. The myofilament arrayed lengthways with directionality. But the sarcomere and the intercalated disk could not be discovered (Figures 1(c)-(e)). After induced by 5-AZA for 4 - 5 weeks, MSCs expressed the specific protein of ANP, PHO, $\beta$-MHC (Figure 1(f)). $\beta$-MHC was expressed much, while the ANPs were little and showed 3 bonds. In the EPCs group, the rate of CD133 expression was $28.8 \% \pm 3 \%$, $17.6 \% \pm 0.9 \%, 11.9 \% \pm 0.4 \%$ and $7.8 \% \pm 1.3 \%$ respectively after the first, second, third and forth week and there was significant difference among these rates (Table 1).

\subsection{The Area of the MI and the Apoptosis}

TTC dying showed the infarction in the Cont group

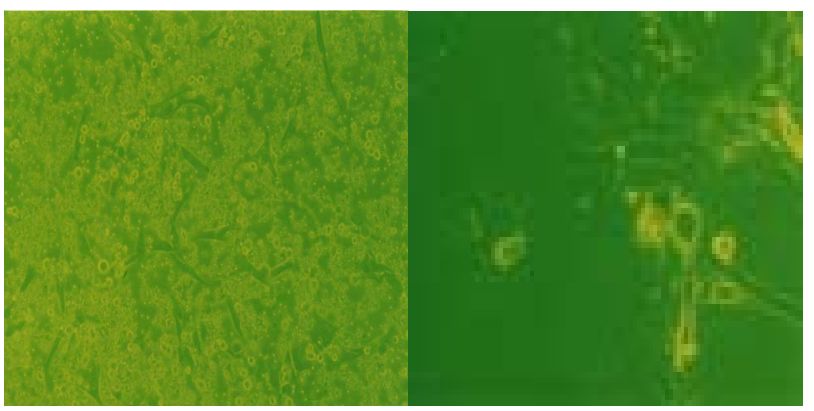

(a)

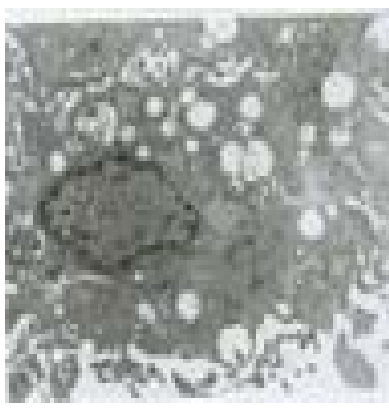

(c)

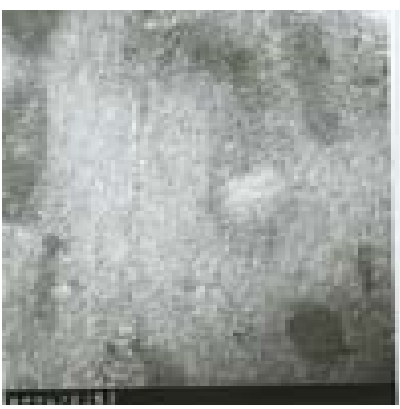

(e) (b)

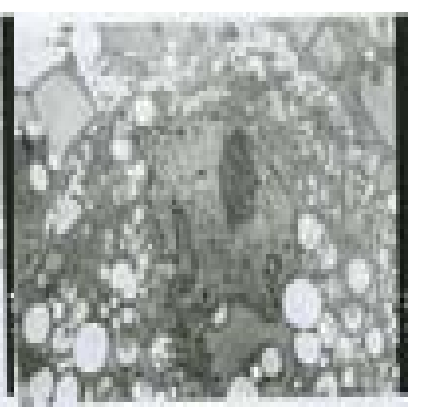

(d)

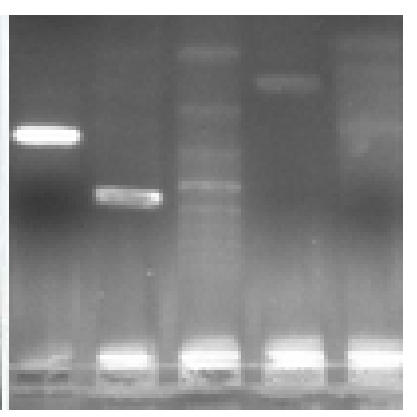

(f)
Figure 1. MSCs were induced and differentiated into CLCs by 5-AZA. (a) After 3 days, there were a few of fusiform-shape cells through phase-contrast microscope $(\times 100)$; (b) After 1 week, there were short stick-like or pearl-like cells through phase-contrast microscope $(\times 100)$; (c) After 2 weeks, the fusiform-shape cells arrayed with directionality through phasecontrast microscope $(\times 100)$; (d) After 4 - 5 weeks, the cell showed one orbicular-ovate nuclear locating in the center by transmission electron microscope $(\times 8000)$; (e) The myofilament arrayed orderly by transmission electron microscope $(\times 8000)$; (f) The results of RT-PCR. From the left to the right is $\beta$-actin, MHC (510000Da), Ladder, PHO (25000Da), ANP (6000Da).

included the the epicardial (Epi) layer, midmyocardial layer and the endocardial (Endo) layer in the anterior wall of left ventricle. However, the infarction in the other 3 groups was limited in or under the the Epi layer (Figure 3). The area of MI was smaller in the cell transplantation groups than in the Cont group and was smallest in the associated cell transplantation group. There was no difference between the CLCs group and the EPCs group (Figure 3). 


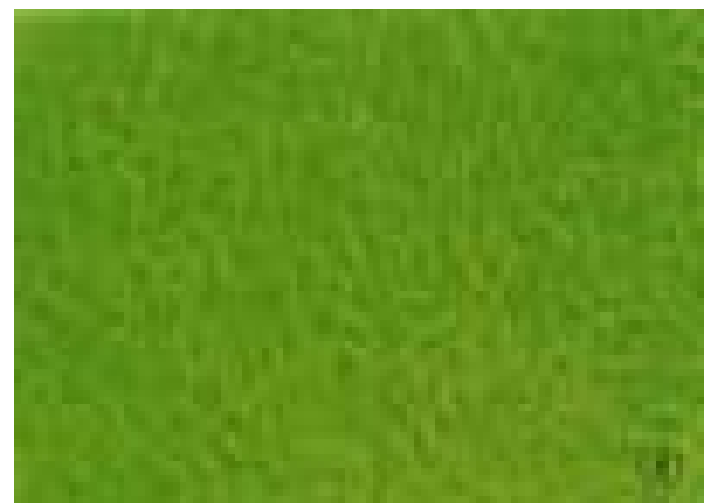

Figure 2. EPCs appeared the typical shape of cobblestone shope or slabstone.

Table 1. The rate of CD133 expression in EPCs at different time.

\begin{tabular}{ccccc}
\hline Time & First week & Second week & Third week & Forth week \\
\hline CD133 (\%) & $28.8 \pm 3$ & $17.6 \pm 0.9^{*}$ & $11.9 \pm 0.4^{*}$ & $7.8 \pm 1.3^{*}$ \\
\hline
\end{tabular}

$*_{P<0.05, \text { compared with each group. }}$
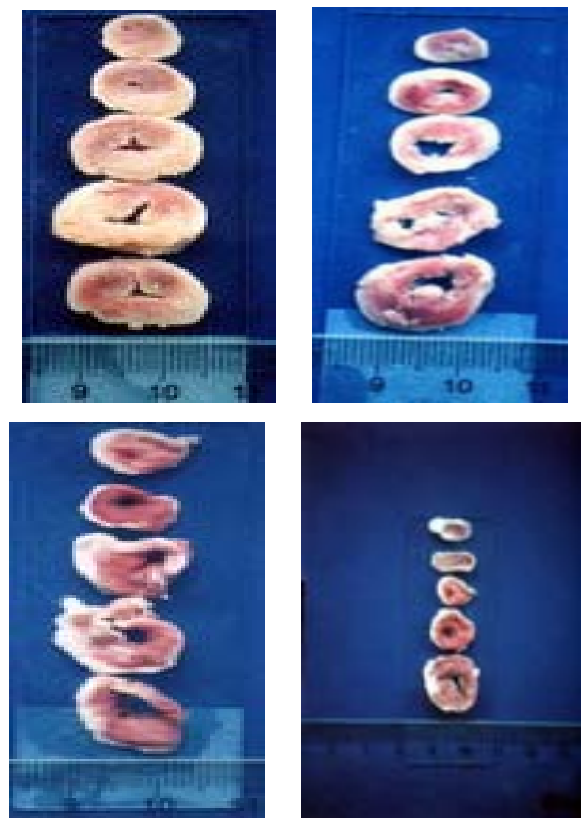

Figure 3. The results of TTC dying.

The pale region showed the infarction. The area of MI from the left to the right is $35.17 \% \pm 0.98 \%$ in the cont group, $28.61 \% \pm 1.24 \%$ in the CLCs group, $29.73 \% \pm$ $2.11 \%$ in the EPCs group and $22.82 \% \pm 3.12 \%$ in the combination group respectively.

\section{DISCUSSION}

It is important to regenerate myocardial cells and repair the necrotic tissues after MI. And it needs the following conditions to perform these: 1 ) the capillary web and the big vessels to transport the oxygen and the nutrition; 2) the myocardial cells to proliferate. So it is necessary for the infarcted myocardium to possess the vascular endothelial cells and reproducible myocardial cells. There are myocardial cells in the heart itself and these cells can differentiate into the mature cells to replace the necrotic cells. But the number of these cells is small, what is more, their function decreases gradually with aging, so they can not improve the heart function sufficiently [3]. There have been lots of studies to testify the characters of mesenchymal stem cells (MSCs). It is believed that MSCs can be induced and differentiate into all kinds of mesenchymal tissues, including cardiomyocycte-like cells (CLCs) and endothelial progenitor cells (EPCs), which can regenerate the myocardial cells and the vessels.

\subsection{Oriented Differentiatinon of the MSCs}

After induced by 5-AZA, MSCs became bigger and appeared obvious myofilament and sarcotubule-like constitution between the cells after 1 week through the phase-contrast microscope. After 10 days, MSCs appeared "short stick" or "pearl" shape and arrayed fusiformly with directionality. Transmission electron microscope showed that the CLCs have one nuclear in the center. They were not binucleate or polynucleate cells and the muscle fibers do not branch. We can distinguish the CLCs from the skeletal muscle cells by these characters [4]. Transmission electron microscope also showed ANPs with the diameter of $200-300 \mu \mathrm{m}$. ANPs locate at the surrounding of the nuclear. They are special ultrastructures of CLCs [4]. Makino [5] studied the short stick-like or pearl-like cells and found that these cells had sarcomere, intercalated disk and the ultrastructure which could beat spontaneously. So he considered these as the characters of CLCs. However, our study did not find these ultrastructures. The possible reasons are: 1 ) the cells in Makino's experiment were immortal cells and they are superior to MSCs, as far as the regeneration and the ability of differentiation were concerned; 2) CLCs are not the mature myocardial cells, but the transitional cells $[6,7] ; 3)$ the culture medium are different too. The fitting internal environment is important to the cells' maturation.

RT-PCR showed that ANPs, PHO, $\beta$-MHC were expressed in CLCs. ANPs is the main component of atrium particle. It has 3 kinds of functional constitutent: $\alpha, \beta, \gamma$, which are not same in the molecular weight and the number of amino acid. So we found 3 bands after electrophoresis. $\beta$-MHC is the main component of Myosin. PHO is sarcoplasmic reticulum binding polypeptide and it can activate the calcium pump to increase absorption and storage of the calcium, which leads the myocardium to relaxation. $\mathrm{PHO}$ and $\beta$-MHC are the special proteins 
of relaxation and contraction. They take part in the performance of the beat, so they are the markers of the ventricular cells.

The above results indicate that MSCs, after induced, have not only the morphous of CLCs but also the special proteins of contraction and relaxation. They are the molecular foundation of the heart function.

Our former experiments had showed that MSCs could be induced and differentiated into EPCs. EPCs appeared like cobblestone shope or slabstone. They can phagocytize the acetylated low density lipoprotein (LDL). Immunohistochemistry showed there were vWF and te lomerase in EPCs. vWF could become the vessels in vitro $[8,9]$. Recent studies proved that the endothelial precursor cells expressed CD133, CD34 and KDR highly; however, CD133 was expressed only in the endothelial ancestral cell, but not in the endothelial cells (ECs). It is generally believed that CD133 was expressed little by little when EPCs differentiate into ECs. So the rate of CD133 expression can be used to distinguish EPCs from ECs [10-13]. In this study, CD133 was highly expressed in the induced MSCs, which indicated that the MSCs could differentiate into endothelial ancestral cells.

At the beginning of induction, CD133 was highly expressed in EPCs. But CD133 decreased dramatically after 2 weeks and it is a single that the EPCs begin to differentiate into the ECs. So it is time to transplant the cells into the infarcted myocardium. What's more, the terminal differentiated ECs can not be transplanted successfully, so it is better to transplant the EPCs in stead of the ECs to regenerate and form the vessels [14]. Considering all of the above reasons, we chose the EPCs to transplant within 2 weeks of induction.

The rate of CD133 expression in our study is different from that of the others. The possible reasons are: 1) the methods to identify and purify the EPSc are not mature and CD133 expresses in the other cells as well; 2) the EPCs are instable. Its phenotype can change and its biologic activity can increase. All of these reasons can influence the results [15-17]; 3) the difference of experiment methods, animal genera and detection time can affect the results too.

\subsection{The Effect of Cell Transplantation on the Myocardial Ischemia}

Not only the singl-cell transplantation but also the associated-cell transplantation can improve the heart function (Table 2), increase the output of each beat, decrease the area of MI, prevent the ventricle remodeling and inhibit the apoptosis (Table 3).

The mechanism about the cell transplantation to treat MI is not very clear. We speculate the possible mechanisms are the following: 1) promoting the myocardial cells and the vessels to regenerate. Compared with the Cont group, the infarction was limited in the epicardial (Epi) layer in the cell transplantation groups, and there was seldom in the midmyocardial (M) layer or the endocardial (Endo) layer. It showed that the area was decreased after cell transplantation. Troponin I and CD34 antigen were expressed highly in the cell transplantation groups. These indicate that the cell transplantation can promote myocardial cells to regenerate and form the vessels in the M layer and the Endo layer. It is beneficial

Table 2. The effect of the cell transplantation on the heart function.

\begin{tabular}{cccccccccc}
\hline & \multicolumn{2}{c}{ The cont group } & \multicolumn{2}{c}{ The CLCs group } & \multicolumn{2}{c}{ The EPCS group } & \multicolumn{2}{c}{ The Comb group } \\
\hline & Pre & Post & Pre & Post & Pre & Post & Pre & Post \\
\hline LVEDD (mm) & $0.99 \pm 0.01$ & $1.53 \pm 0.13$ & $1.49 \pm 0.11$ & $1.24 \pm 0.02^{*}$ & $1.35 \pm 0.14$ & $1.21 \pm 0.09^{*}$ & $1.51 \pm 0.23$ & $1.01 \pm 0.01^{*}$ \\
LVESD (mm) & $0.62 \pm 0.32$ & $1.23 \pm 0.02$ & $1.08 \pm 0.09$ & $0.98 \pm 0.04^{*}$ & $1.03 \pm 0.17$ & $0.98 \pm 0.12^{*}$ & $1.37 \pm 0.19$ & $1.11 \pm 0.03^{*}$ \\
LVPWH (cm) & $0.21 \pm 0.01$ & $0.23 \pm 0.02$ & $0.27 \pm 0.03$ & $0.24 \pm 0.02$ & $0.23 \pm 0.01$ & $0.21 \pm 0.01$ & $0.28 \pm 0.04$ & $0.23 \pm 0.03$ \\
FS (\%) & $37.51 \pm 1.21$ & $13.8 \pm 0.36$ & $27.43 \pm 2.11$ & $27.38 \pm 3.12^{*}$ & $23.68 \pm 1.25$ & $26.98 \pm 2.19^{*}$ & $20.62 \pm 1.37$ & $32.12 \pm 3.41^{*}$ \\
SV (ml) & $2.13 \pm 0.02$ & $1.93 \pm 0.02$ & $1.97 \pm 0.12$ & $2.13 \pm 0.09^{*}$ & $2.43 \pm 0.11$ & $3.40 \pm 0.21^{*}$ & $2.64 \pm 0.32$ & $3.28 \pm 0.21^{*}$ \\
LVEF (\%) & $52.58 \pm 3.12$ & $32.49 \pm 1.29$ & $49.67 \pm 1.24$ & $53.22 \pm 2.13^{*}$ & $51.42 \pm 2.31$ & $56.91 \pm 2 . .04^{*}$ & $54.27 \pm 1.29$ & $62.61 \pm 2.37^{*}$ \\
\hline
\end{tabular}

Pre means pre-transplantation; Post means post-transplantation; LVEDD means the left ventricular end diastolic diameter; LVESD means the left ventricular end systole diameter; LVPWH means the left ventricular posterior wall hypertrophy; FS means the fractional shortening; SV means the stork volume; LVEF means the left ventricular ejection fraction. ${ }^{*} P<0.05$, post vs pre.

Table 3. The rate of necrosis in each group.

\begin{tabular}{ccccc}
\hline Group & The Cont group & The CLCs group & The EPCs group & The Comb group \\
\hline Necrosis (\%) & $8.6 \pm 0.94$ & $6.94 \pm 0.59 \%$ & $6.4 \pm 0.27^{*}$ & $4.63 \pm 0.74 \%$ \\
\hline * & & & \\
\hline
\end{tabular}

${ }^{*} P<0.05$, the cont group vs the cell transplantation groups; ${ }^{*} P<0.05$, the associated cell transplantation vs the single cell transplantation. 
for enhancing the contraction and left ventricular ejection fraction. Luciano injected the self stem cells into cardiac muscle of female Yorkshire pigs whose left anterior descending had been ligated [18]. He found that myocardial cells regenerated between the EPi layer and the Endo layer and the cells regenerated more than those in the control. Immunohistochemistry showed the regeneration-relative antigens, such as Ki-67 and c-kit, were expressed significantly in the cells. His experiment indicated that the cell regeneration is the main mechanism of the cell transplantation to treat MI; 2) inhibiting the apoptosis. This study shows the cell transplantation can inhibit the apoptosis and this is an important aspect of the cell transplantation. It is probably relative to the stem cell factor (SCF). Firstly, the myocardium interstitium fibroses and the inflammation take place after MI. Fibroblast and macrophage express SCF. MSCs have c-kit expressed at its surface, which is the ligand of SCF. When MSCs have been transplanted into the infarcted myocardium, c-kit connects with SCF and the latter can inhibit PIK3 and regulate phospholipase C (PPC) and apoptosis activated by PPC [19-21]. Secondly, the necrotic tissues can cause inflammation, tumor necrosis factor (TNF), transforming growth factor- $\beta 1$ (TGF- $\beta 1$ ). These factors can induce apoptosis receptors, such as Fas, TNF superfamily and TGF- $\beta$ receptor, et al. These receptors connect with relevant ligands and activate the apoptosis induced by caspases superfamily. What's more, SCF can inhibit the apoptosis induced by TNF and TGF- $\beta 1$ [22-24]; 3 ) the number of the transplanted cells. If the MSCs were induced and differentiated into CLCs and EPCs in vivo, the number of CLCs and EPCs is small and it will reduce the curative effect [25]. We can increase the number of the cells by inducing MSCs in vitro. And it is benefit to concentrate the cells by injecting them into the surrounding of the infarcted myocardium. This method is feasible in our experiment; 4) interfering in the ventricle remodeling. This study indicates that the heart function has been improved after the cell transplantation, such as, the LVEDD and the LVESD shortening, the output of each beat increasing, and so on. It is probably because the cell transplantation interferes in the ventricle remodeling. Its possible mechanism is that the cell transplantation inhibits the myocardium interstitial fibrosis. The researchers [26] transplanted the MSCs into the infarced myocardium of the rats and detected the expression of collagen I, II, matrix metalloproteinase and TGF- $\beta 1$ by the means of immunohistochemistry, RT-PCR and in-situ hybridization. After one month, they found that the concentration of collagen I, II, matrix metalloproteinase and TGF- $\beta 1$ decreased significantly in the MSCs group, however, extracellular matrix and TGF- $\beta 1$ increased in the MI control group. TGF- $\beta 1$ is the most important factor which can cause myocar- dium interstitial fibrosis so far. It can regulate the synthesis and the decomposition of extracellular matrix and promote the fibroblast regeneration. So the cell transplantation can prevent ventricle remodeling, but the thickness of the ventricle wall is not different between the Cont group and the cell transplantation group. It is probably because of the short time of observation [27].

In a conclusion, the heart function in the Cont group worsened gradually after MI. However, the heart function and the ventricle remodeling improved in the cell transplantation groups. Compared among the cell transplantation groups, the associated cell transplantation is superior to the single cell transplantation at the aspects of the heart function, necrosis and the area of MI. There is no difference between the CLCs group and the EPCs group considering the curative effect. Maybe it is because the CLCs regenerate the myocardial cells and increase the contraction, but do not improve the blood flow [28]. While the EPCs form the vessels and enhance the perfusion, but do not increase the contraction. The associated cell transplantation offer both the CLCs and the EPCs, so it can improve the contraction and the perfusion. It is better for the transplanted cells to survive and the hibernating myocardial cells and the stunning myocardial cells to recover. What's more, after the MSCs are induced, the receptors and the ligands at their surface change. They can react with some cytokine and inflammatory factors and take positive action in the inflammatory reaction, antagonize ischemia and hypoxia and improve the apoptosis caused by oxygen-derived free radicals [29]. And these effections are better in the associated cell transplantation than in the single cell transplantation. After the associated cell transplantation, CLCs and EPCs react with each other and lead EPCs to differentiate into the active myocardial cells, so it increases the number of the myocardial cells. It is more benefit to improve the heart contraction [30].

There are limitations in our study. Firstly, MSC can not differerntiate into CLCs abundantly very much after induced by 5-AZA. Secondly, it is not enough to determine the time of the EPCs transplantation by the concentratin of CD133. Thirdly, the cells have not been purified after induced and differentiated orientatedly [31].

\section{REFERENCES}

[1] Flynn, A., Chen, X., O'Connell, E., et al. ( 2012) A comparison of the efficacy of transplantation of bone marrowderived mesenchymal stem cells and unrestricted somatic stem cells on outcome after acute myocardial infarction. Stem Cell Research \& Therapy, 3, 36-44. http://dx.doi.org/10.1186/scrt127

[2] Zeinaloo, A., Zanjani, K.S., Bagheri, M., et al. (2011) Intracoronary administration of autologous mesenchymal stem cells in a critically ill patient with dilated cardio- 
myopathy. Pediatric Transplantation, 15, E183-E186. http://dx.doi.org/10.1111/j.1399-3046.2010.01366.x

[3] Maureira, P., Marie, P.Y., Yu, F., et al. (2012) Repairing chronic myocardial infarction with autologous mesenchymal stem cells engineered tissue in rat promotes angiogenesis and limits ventricular remodeling. Journal of Biomedical Science, 19, 93-99. http://dx.doi.org/10.1186/1423-0127-19-93

[4] Kim, S.W., Lee, D.W., Yu, L.H., et al. (2012) Mesenchymal stem cells overexpressing GCP-2 improve heart function through enhanced angiogenic properties in a myocardial infarction model. Cardiovascular Research, 95, 495-506. http://dx.doi.org/10.1093/cvr/cvs224

[5] Kim, S.W., Lee, D.W., Yu, L.H., et al. (2012) Mesenchymal stem cells overexpressing GCP-2 improve heart function through enhanced angiogenic properties in a myocardial infarction model. Cardiovascular Research, 95, 495-506. http://dx.doi.org/10.1093/cvr/cvs224

[6] Chi, N.H., Yang, M.C., Chung, T., et al. (2012) Cardiac repair achieved by bone marrow mesenchymal stem cells/ silk fibroin/hyaluronic acid patches in a rat of myocardial infarction model. Biomaterials, 33, 5541-5551. http://dx.doi.org/10.1016/j.biomaterials.2012.04.030

[7] Wang, T., Sun, S., Wan, Z., et al. (2012) Effects of bone marrow mesenchymal stem cells in a rat model of myocardial infarction. Resuscitation, 83, 1391-1396. http://dx.doi.org/10.1016/j.resuscitation.2012.02.033

[8] Zheng, S.X., Weng, Y., Zhou, C., et al. (2012) Comparison of cardiac stem cells and mesenchymal stem cells transplantation on the cardiac electrophysiology in rats with myocardial infarction. Stem Cell Reviews, 4, 1-11.

[9] Otto Beitnes, J., Oie, E., Shahdadfar, A., et al. (2012) Intramyocardial injections of human mesenchymal stem cells following acute myocardial infarction modulate scar formation and improve left ventricular function. Cell Transplant, 21, 1697-1709. http://dx.doi.org/10.3727/096368911X627462

[10] Du, Y.Y., Yao, R., Hu, X.Q., et al. (2011) Dural modulation effects of mesenchymal stem cells implantation on myocardial collagen remodeling in a rat model of myocardial infarction. Chinese Journal of Cardiovascular Diseases, 39, 840-846.

[11] Xie, X., Sun, A., Zhu, W., et al. (2012) Transplantation of mesenchymal stem cells preconditioned with hydrogen sulfide enhances repair of myocardial infarction in rats. Tohoku Journal of Experimental Medicine, 226, 29-36. http://dx.doi.org/10.1620/tjem.226.29

[12] Shi, B., Liu, Z.J., Zhao, R.Z., et al. (2011) Effect of mesenchymal stem cells on cardiac function and restenosis of injured artery after myocardial infarction. National Medical Journal of China, 91, 2269-2273.

[13] Mishra, P.K. (2008) Bone marrow-derived mesenchymal stem cells for treatment of heart failure: Is it all paracrine actions and immunomodulation? Journal of Cardiovascular Medicine, 9, 122-128. http://dx.doi.org/10.2459/JCM.0b013e32820588f0

[14] Du, Y.Y., Yao, R., Pu, S., et al. (2012) Mesenchymal stem cells implantation increases the myofibroblasts congergating in infarct region in a rat model of myocardial in- farction. Chinese Journal of Cardiovascular Diseases, 40 , 1045-1050.

[15] Wen, Z., Zheng, S., Zhou, C., et al. (2012) Bone marrow mesenchymal stem cells for post-myocardial infarction cardiac repair: microRNAs as novel regulators. Journal of Cellular and Molecular Medicine, 16, 657-671. http://dx.doi.org/10.1111/j.1582-4934.2011.01471.x

[16] Nayan, M., Paul, A., Chen, G., et al. (2011) Superior therapeutic potential of young bone marrow mesenchymal stem cells by direct intramyocardial delivery in aged recipients with acute myocardial infarction: In vitro and in vivo investigation. Journal of Tissue Engineering, 11, 211-223.

[17] Jin, J., Zhao, Y., Tan, X., et al. (2011) An improved transplantation strategy for mouse mesenchymal stem cells in an acute myocardial infarction model. PLoS One, 6, e21005. http://dx.doi.org/10.1371/journal.pone.0021005

[18] Vassalli, G. and Moccetti, T. (2011) Cardiac repair with allogeneic mesenchymal stem cells after myocardial infarction. Swiss Medical Weekly, 141, w13209.

[19] Carlson, S., Trial, J., Soeller, C., et al. (2011) Cardiac mesenchymal stem cells contribute to scar formation after myocardial infarction. Cardiovascular Research, 91, 99107. http://dx.doi.org/10.1093/cvr/cvr061

[20] Wen, Z., Zheng, S., Zhou, C., et al. (2011) Repair mechanisms of bone marrow mesenchymal stem cells in myocardial infarction. Journal of Cellular and Molecular Medicine, 15, 1032-1043.

http://dx.doi.org/10.1111/j.1582-4934.2010.01255.x

[21] Armiñán, A., Gandía, C., García-Verdugo, J.M., et al. (2010) Mesenchymal stem cells provide better results than hematopoietic precursors for the treatment of myocardial infarction. Journal of the American College of Cardiology, 55, 2244-2053. http://dx.doi.org/10.1016/j.jacc.2009.08.092

[22] Hare, J.M., Traverse, J.H., Henry, T.D., et al. (2009) A randomized, double-blind, placebo-controlled, dose-escalation study of intravenous adult human mesenchymal stem cells (prochymal) after acute myocardial infarction. Journal of the American College of Cardiology, 54, 22772086. http://dx.doi.org/10.1016/i.jacc.2009.06.055

[23] Li, Z., Guo, J., Chang, Q., et al. (2009) Paracrine role for mesenchymal stem cells in acute myocardial infarction. Biological \& Pharmaceutical Bulletin, 32, 1343-1346. http://dx.doi.org/10.1248/bpb.32.1343

[24] Mias, C., Lairez, O., Trouche, E., et al. (2009) Mesenchymal stem cells promote matrix metalloproteinase secretion by cardiac fibroblasts and reduce cardiac ventricular fibrosis after myocardial infarction. Stem Cells, 27, 2734-2743. http://dx.doi.org/10.1002/stem.169

[25] Grauss, R.W., Winter, E.M., van Tuyn, J., et al. (2007) Mesenchymal stem cells from ischemic heart disease patients improve left ventricular function after acute myocardial infarction. American Journal of Physiology, Heart and Circulatory Physiology, 293, H2438-H2447. http://dx.doi.org/10.1152/ajpheart.00365.2007

[26] Berger, S., Aronson, D., Lavie, P., et al. (2013) Endothelial progenitor cells in acute myocardial infarction and sleep-disordered breathing. American Journal of Respi- 
ratory and Critical Care Medicine, 187, 90-98. http://dx.doi.org/10.1164/rccm.201206-1144OC

[27] Kuliczkowski, W., Derzhko, R., Prajs, I., et al. (2012) Endothelial progenitor cells and left ventricle function in patients with acute myocardial infarction: Potential therapeutic considertions. American Journal of Therapeutics, 19, 44-50. http://dx.doi.org/10.1097/MJT.0b013e3181e0cab3

[28] Schuh, A., Liehn, E.A., Sasse, A., et al. (2010) Transplantation of endothelial progenitor cells improves neovascularization and left ventricular function after myocardial infarction in a rat model. Basic Research in Cardiology, 103, 69-77.

http://dx.doi.org/10.1007/s00395-007-0685-9

[29] Lev, E.I., Kleiman, N.S., Birnbaum, Y., et al. (2005) Cir-

\section{ABBREVIATIONS}

MSC: mesenchymal stem cell

MI: myocardial infarction

EPC: endothelial progenitor cells

CLCs: cardiomyocycte-like cells

ECGS: endothelial cell growth supplements

LAD: left anterior descending

$\beta$-MHC: $\beta$-myosin heavy main

ECs: endothelial cells

PHO: phospholamban

ANP: atrial natriuretic polypeptide culating endothelial progenitor cells and coronary collaterals in patients with non-ST segment elevation myocardial infarction. Journal of Vascular Research, 42, 408414. http://dx.doi.org/10.1159/000087370

[30] Mitchell, A.J., Sabondjian, E., Blackwood, K.J., et al. (2013) Comparison of the myocardial clearance of endothelial progenitor cells injected early versus late into reperfused or sustained occlusion myocardial infarction. International Journal of Cardiovascular Imaging, 29, 497-504. http://dx.doi.org/10.1007/s10554-012-0086-5

[31] Jung, C., Florvaag, A., Oberle, V., et al. (2012) Positive effect of eplerenone treatment on endothelial progenitor cells in patients with chronic heart failure. Journal of the Renin-Angiotensin-Aldosterone System, 13, 401-406. http://dx.doi.org/10.1177/1470320312447650

LVESD: left ventricular end systole diameter LVEDD: the left ventricular end diastolic diameter LVPWH: the left ventricular posterior wall hypertrophy FS: the fractional shortening

SV: the stork volume

LVEF: left ventricular ejection fractio

Cont group: the control group

CLC group: the cardiomyocycte-like cell group EPC group: the endothelial progenitor cell group Comb group: the combination group 